https://doi.org/10.22519/22157360.893

\title{
El clima organizacional aspecto estrategico en el servicio al \\ cliente
}

\section{The organizational climate strategic aspect in customer \\ service}

Yuleimy Ortega Ruiz ${ }^{1}$

Lino Mercado León ${ }^{2}$

\section{Resumen}

El presente artículo tiene como objetivo analizar el clima organizacional y el servicio al cliente en las empresas de servicio. Debido a que la calidad en el servicio que se les da a los clientes, se instituye como el factor diferenciador, permitiendo dar un valor agregado a la organización frente a otras en el mercado. El servicio al cliente y el clima organizacional que se viva en una organización debe ser comprendida como una estrategia en la que toda la empresa debe de estar enfocada. Resulta indispensable entonces, contar con personal idóneo y capacitado para satisfacer las necesidades y expectativas de los grupos de interés relacionados con la organización.

Palabras clave: Clima organizacional; servicio al cliente; organizaciones empresariales; motivación; planeación estratégica.

\footnotetext{
${ }^{1}$ Administradora de Empresas. Universidad Los Libertadores yuleimiortega@gmail.com
}

AGLALA ISNN 2215-7360

2014; 5 (1): 126-142 
$2 \quad$ Administrador de Empresas. Especialista en Gerencia de Mercadeo

Docente en la Fundación Universitaria Tecnológico Comfenalco linomercado@gmail.com CÓDIGO JEL: M19

Fecha de recepción: Octubre de 2014 / Fecha de aceptación en forma revisada: Diciembre 2014

\begin{abstract}
This paper aimed to analyze the organizational climate and customer service in business service. Because the quality of service they provide to clients is the factor that is the differentiator and gives a high value to the organization from others in the market, the service to customer and organizational climate to be alive in an organization must be understood as a strategy in which the whole company must be focused. It is essential then, have qualified and trained staff to meet the needs and expectations of stakeholders related to the organization.
\end{abstract}

Keywords: Organizational climate; customer service; business organizations; motivation; strategic planning.

\title{
Introducción
}

El clima laboral aparece en circunstancias en que se hacía necesario tener presente los fenómenos globales que se presentaron en las organizaciones, desde una perspectiva holística, pero a la vez, lo suficientemente sencillo para que sirva de orientación a investigaciones donde se puedan generar practicidades de intervención en las empresas.

Al clima laboral se le puede denominar desde diversas concepciones, tales como ambiente laboral, el entorno laboral, ambiente de trabajo. Fue en el año de 1960 cuando Gellerman (citado en Brunet, 1987), acuñó el término por primera vez. Este autor expresó que el clima es la AGLALA ISNN 2215-7360

2014; 5 (1): 126-142 
convergencia de dos grandes escuelas de la psicología como son: La Gestalt, que ve cómo la organización influye en el comportamiento de las personas y, la Funcionalista, en la que el comportamiento está directamente relacionado con las diferencias individuales y el ambiente en una organización.

Las empresas sin llevar a menos su tamaño o razón social, han entendido que el capital monetario, financiero o tecnológico no es suficiente para conseguir ventajas competitivas frente a la competencia. Para esto requieren contar con un ambiente idóneo, personal calificado y competente que responda rápidamente al cambio y sea capaz de satisfacer las necesidades de los clientes, permitiéndole así alcanzar sus objetivos.

La importancia de conocer el clima organizacional basa su fundamento en la influencia que ejerce sobre el comportamiento de los trabajadores, siendo vital su diagnóstico que permita el diseño de estrategias empresariales.

El trabajo realizado busca enfatizar sobre el clima organizacional y su influencia en el servicio al cliente.

El método de estudio es cualitativo y de tipo exploratorio y las fuentes de recolección de la información fueron las secundarias, ya que se utilizaron en la consulta libros, revistas y base de datos y la técnica que se desarrolló fue el análisis documental.

\section{Clima organizacional en las organizaciones empresariales}

Para Snow (2003), el clima organizacional es la apreciación de la forma en que se siente trabajar en un determinado ambiente. Es la atmósfera que se presenta en un lugar de trabajo y está caracterizado por una mixtura compleja de normas, valores, expectativas, políticas y procedimientos que son de influencia para las formas conductuales, tanto individuales como de AGLALA ISNN 2215-7360

2014; 5 (1): 126-142 
grupo. Esto permite afirmar que son los administradores los responsables de primera línea de crear el clima en todas las áreas de la organización. Al fluir un clima positivo se creará un lugar fácil y de mucha comodidad para laborar y por ende, los trabajadores estarán más satisfechos por su trabajo y, más comprometidos con la empresa.

En ese mismo sentido, Cohen (1992), expresa que la cultura puede conceptualizarse como "la suma total de los rasgos de comportamiento y de las creencias, que son características aprendidas, de los miembros de una sociedad en particular" (p. . Ante esto, cabe resaltar que cuando se habla de comportamiento y creencias, estos se manifiestan a través de los valores, costumbres y tradiciones, se refiere a un sistema de significados que son compartidos por una gran parte de los individuos de una organización y que permite distinguirlas entre otras, con lo cual se puede inferir que el clima laboral está dentro del estudio de la cultura organizacional, es decir forma parte de ella.

La cultura organizacional es la manera de pensamiento y de acción que tiene una organización, lo cual es fruto de las interrelaciones del pasado y presente para poderse adaptar a contextos del ambiente y de las resistencias internas (Schein, 1996). Además, en la cultura de la empresa se da la intervención colectiva y que por medio de un periodo de tiempo se desarrollan las acciones, las políticas y decisiones, como también un gradual establecimiento de procederes tanto formales como informales. Por lo tanto, se debe de reconocer que existen mecanismos que desarrollan y consolidan la cultura dentro de una entidad y que se manifiestan a través de rituales, símbolos, transmisión de mitos y leyendas, lenguajes propios y sistemas de recompensas monetarias y no monetarias. Ante esto, se puede decir que la cultura organizacional es el conjunto de los valores, las creencias y entendimientos que en común tienen los que integran una 
organización. Es además, un sistema de significados que son compartidos por una gran parte de los individuos de una empresa y que dan diferencia entre una y otra.

La cultura organizacional se presenta como la cultura dominante dentro de una organización y son los valores centrales que comparte la mayoría de los integrantes de un organismo empresarial.

Desde la perspectiva psicológica, (Pelaes, 2010), expresa que el clima se puede analizar desde dos puntos de vista a tratar: a) Las características que se dan en el ambiente de un grupo humano expresa al conjunto de aspectos psicológicos, tales como el liderazgo, la motivación, etc., que se dan entre los que integran una organización y que tiene relación con lo afectivo, cognitivo y de carácter de cada individuo; sin embargo, para analizar estos procesos se lleva a cabo mediante las dimensiones del clima organizacional. b) La influencia del clima organizacional en la conducta de los trabajadores es básicamente una síntesis de actividades, debido a que desde la perspectiva psicológica de las empresas, todas las acciones que ejecutan los trabajadores son conductas, por lo tanto tienen su base en los elementos personales de sus individuos.

De forma similar, Seisdedos en el año 1996 da una conceptualización sobre el clima organizacional al expresar que es el conjunto de percepción global, tanto personal como psicológico, que el sujeto tiene de la organización y que tal concepción se debe también por la interacción entre el individuo y la empresa. Lo importante es la forma como la persona percibe su entorno sin tomar en cuenta la percepción de otra, por ello se puede decir que el clima es una dimensión que proviene del sujeto más que de la organización en sí. Otro autor, Sandoval (2004) refuerza el aspecto individual al considerar que el clima es la percepción que los empleados y directivos se forman de una entidad a la que pertenecen y, por lo tanto, determina el desempeño de la organización.

AGLALA ISNN 2215-7360

2014; 5 (1): 126-142 
Por consiguiente, se puede presentar al clima laboral como un eje de dos coordenadas. Esto debido a que hay autores que sitúan el clima como real y externo al sujeto; en cambio, hay otros que acentúan la dimensión psicológica o subjetiva. Aunque hay autores que intentan dar un equilibrio a ambos enfoques.

El modelo de Clima Laboral de Trickett y Moss (citado en Tarazona y Roger, 2004), convienen en afirmar que el clima es un concepto sistémico y que en cada organización se presenta de manera única debido a que posee su propia cultura, tradiciones y formas de acción que sumadas dan el clima organizacional y que por medio de ella influye en el desempeño, motivación y satisfacción en un puesto laboral. Como sistema, el clima laboral manifiesta el tipo de vida de una organización en su totalidad. Ahora, si se mejora el clima, pues se tendrá una mejoría en el desempeño de los trabajadores.

Según Bustos y Miranda (2001), al exponer sobre el clima organizacional, se basan en que el tal presenta características, tales como: En primer lugar, las características del medio ambiente en la organización; estas son tanto internas como externas. Además, son percibidas de manera directa o indirecta por los individuos que realizan sus labores en ese medio ambiente. Debido a ello, cada empleado percibe el medio en que se desenvuelve de manera distinta. En segundo lugar, el clima en una empresa se presenta a través de cambios temporales en las actitudes de las personas y que se pueden dar debido a diversas razones, tales como cuando se presenta una reducción de personal, cuando se dan incrementos salariales, etc. Cuando en una organización se aumenta la motivación esta repercutirá en un aumento del clima laboral y es observable por las ganas de trabajar. En cambio, cuando se presenta una disminución en la motivación, se dará un descenso en la ganas de laborar. Además, hay que tener presente que esto se puede presentar de diferentes maneras entre unas y otras empresas o entre unas y otras secciones dentro de una misma institución. AGLALA ISNN 2215-7360

2014; 5 (1): 126-142 
En último lugar, el clima es un sistema interdependiente donde se presentan un dinamismo constante, a través de su estructura, sus características organizacionales y de las personas que forman parte de ella.

Kolb en el año 2001, por medio de varias investigaciones sobre comportamiento organizacional, observó la importancia de los factores liderazgo, motivación y clima laboral, por lo cual se presenta una relación directa entre liderazgo y clima organizacional, se da una asociación entre una forma de clima laboral y una motivación determinada hacia la labor y, por último, el clima que se da en una organización se asocia a los cambios en los rasgos de conducta que son en apariencia estables.

Likert, (citado en Brunet, 2007), da una clasificación del clima laboral de acuerdo a las siguientes características:

\begin{tabular}{|c|c|c|c|}
\hline \multicolumn{2}{|c|}{ Cllma de flpo autoritarlo } & \multicolumn{2}{|c|}{ Cllma de flipo parflcipativo } \\
\hline $\begin{array}{c}\text { Sistema I- Autoritarismo } \\
\text { explotador }\end{array}$ & $\begin{array}{c}\text { Sistema II-Autoritarismo } \\
\text { paternalista }\end{array}$ & Sistema III -Consultivo & $\begin{array}{l}\text { Sistema IV - Participativo } \\
\text { en grupo }\end{array}$ \\
\hline 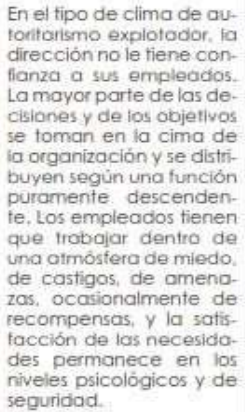 & $\begin{array}{l}\text { 日 tipo de clima autoritarls- } \\
\text { mo patemalista es aquel } \\
\text { en que la dirección tiene } \\
\text { una confianza condescen- } \\
\text { diente en sus empleados, } \\
\text { como la de un amo con } \\
\text { su siervo. La mayor parte } \\
\text { de las decisiones se toman } \\
\text { en la cima, pero algunas se } \\
\text { toman en los escaiones in- } \\
\text { feriores, Las recompensos y } \\
\text { algunas veces los castigos } \\
\text { son los métodos utilizados } \\
\text { por excelencia para mots- } \\
\text { var a los trabajadores. }\end{array}$ & $\begin{array}{l}\text { La dirección que evolucio- } \\
\text { na dentro de un clima par- } \\
\text { ticipativo tiene confanza } \\
\text { en sus empleados. La po- } \\
\text { litica y las decisiones se to- } \\
\text { man generalmente en la } \\
\text { cima pero se permite a los } \\
\text { subardinados que fomen } \\
\text { decisiones más especificas } \\
\text { en los niveles inferiores. La } \\
\text { comunicación es de tipo } \\
\text { descendente. Las recom- } \\
\text { pensas, los castigos oca- } \\
\text { sionales y cualquier im- } \\
\text { picación se utilizan para } \\
\text { motivar a los trabajadores: } \\
\text { se trata tambien de satis- } \\
\text { lacer sus necesiododes de } \\
\text { prestigio y de estima. }\end{array}$ & $\begin{array}{l}\text { En el sistema de la participa- } \\
\text { ción en grupo, lo dirección } \\
\text { fiene plena confianza en sus } \\
\text { empleados. Los procesos de } \\
\text { toma de decisiones están di- } \\
\text { seminados en foda la organi- } \\
\text { zación, y muy bien integrados } \\
\text { a cada uno de los niveles. La } \\
\text { comunicación no se hace } \\
\text { solamente de manera ascen- } \\
\text { dente a descendente. sino } \\
\text { también de forma laterat. Los } \\
\text { empleados están motivados } \\
\text { por la participación y la impi- } \\
\text { cación, por el establecimien- } \\
\text { to de objetivos de rendimien- } \\
\text { to. por et mejotamiento de los } \\
\text { metodos de trabajo y por la } \\
\text { evaluación del rendimiento } \\
\text { en función de los objetivos. }\end{array}$ \\
\hline
\end{tabular}

Figura 1. Clasificación del clima laboral

Lo que se puede desprender de esta figura es que cada vez que el clima de una organización esté más cerca al sistema IV Participativo en grupo, las relaciones entre la gerencia y el personal serán mejores; por el contrario, cuando el clima se aproxime más al sistema I Autoritarismo explotador, esta será menos buena.

AGLALA ISNN 2215-7360

2014; 5 (1): 126-142 
Por otro lado, este autor indica que existen tres variables que determinan a la organización desde el punto de vista del clima que son: Primero, las variables causales o independientes, que indican la manera en que una organización evoluciona y consigue sus resultados y se presenta a través de la estructura organizativa, las reglas, las decisiones, las competencias y actitudes. Segundo, las variables intermedias, que indican el estado interno y de salud de una entidad, estos son las actitudes, los objetivos, la comunicación eficaz, la toma de decisiones y la motivación. Tercero, las variables finales o dependientes, que son el resultado producto de lo que se presentan en las variables independientes e intermedias. Estas reflejan los logros que la institución logró conseguir tales como la productividad, los gastos, las ganancias y pérdidas.

Para poder efectuar estudios sobre el clima en una organización, es determinante entender las variables ambientales que repercuten en el comportamiento y la productividad de los individuos que forman parte de una institución (Pfeffer, 2006). Este autor expone los siguientes: Ambiente físico, espacio físico, condiciones de ruido, calor y contaminación; estructura, tamaño de la organización, estructura formal, estilo de dirección, ambiente social, compañerismo, conflictos interpersonales, comunicación, personal, aptitudes y actitudes, motivación y expectativas, comportamiento organizacional, productividad, ausentismo y rotación de personal, satisfacción laboral, tensiones y estrés. Es a través de estas variables que se puede llevar a cabo un diagnóstico del clima en una empresa.

Para llevar a cabo el diagnóstico del clima organizaciónal en las empresas, se pueden utilizar las dimensiones que Litwin y Stinger (citado en Kolb y Mcintyre, 2001), proponen que se pueden utilizar para poder explicar el clima que se da en una organización. Estas son:

AGLALA ISNN 2215-7360

2014; 5 (1): 126-142 
1. Estructura. Que representa la apreciación que los miembros de una organización tienen en lo concerniente a las reglas, procedimientos, tramitaciones y otras, que afectan el desarrollo laboral.

2. Responsabilidad. Es el sentir que los miembros de la institución tienen en lo referente a su autonomía para la toma de decisiones en sus puestos laborales. Estos son sujetos a supervisión general y no estrecha, lo que les da a los trabajadores cierta autonomía de decisión.

3. Recompensa. Tiene que ver con la percepción que tienen los integrantes de la empresa en lo referente al buen trabajo y si es que se utiliza más el premio que el castigo.

4. Desafío. Se refiere a los sentimientos que tienen los trabajadores de una organización acerca de los desafíos que trae el trabajo. Además, tiene que ver cuando la institución asume riesgos calculados a fin de conseguir los objetivos organizacionales.

5. Relaciones. Es el ambiente de trabajo agradable que es percibido por los trabajadores, así como las buenas interrelaciones sociales que se dan entre pares, así como entre jefes y subordinados.

6. Cooperación. Es el sentir que tienen los empleados ante la ayuda mutua que existe entre directivos y trabajadores, tanto en un departamento como entre departamentos.

7. Estándares. Tiene que ver con el énfasis que tiene una organización por el rendimiento de los trabajadores.

8. Conflicto. Es cuando se aceptan los puntos de vista discrepantes y no se tiene temor a enfrentar y buscar soluciones ante los problemas que se presenten en la organización.

9. Identidad. Es el sentimiento de pertenencia a la institución y es de vital importancia entre los trabajadores.

AGLALA ISNN 2215-7360

2014; 5 (1): 126-142 
Por otro lado, aunque se puede conseguir cambios en el clima en la organización por medio de políticas o decisiones eficaces, es complicado conseguir que el clima no regrese a su anterior momento cuando han desaparecido los efectos de medidas adoptadas. Esto se puede explicar a través de un ejemplo en el cual una organización en cuyo interior existe descontento generalizado, se puede conseguir mejorar la situación por medio de la promesa de un aumento salarial. Sin embargo, tal efecto puede quedar anulado si no se llevan a cabo cambios que logren llevar a la empresa a otra situación. Esto podría generar actitudes de desesperanza y fuertemente negativas de los trabajadores hacia el organismo y que esto al final afecta el clima organizacional.

\section{Servicio al cliente en las organizaciones empresariales}

El cliente es el individuo u organización que adquiere de manera libre y voluntaria productos o servicios que desea para sí o para otra persona y, en definitiva, es la razón principal por la cual se crean, producen y comercializan bienes o servicios.

Los clientes tienen expectativas y se traduce al nivel de servicio que un cliente desea recibir. De acuerdo con Rust, Zahorick y Keiningham (citado en Koenes, 1996), exponen tres niveles a saber: a) Las expectativas ideales, que son los que el cliente espera que suceda y de esa forma se ha satisfecho sus necesidades y expectativas que se generan al adquirir un bien o un servicio. b) Expectativas de lo que debería ser, es cuando el cliente considera lo que debería recibir según su pago ante un bien o servicio. c) Expectativas esperadas, es lo que los clientes esperan, debido a que por medio de otras personas son informadas por los productos o servicios de una organización y van a calificarlas según el servicio que el usuario recibió.

El servicio al cliente es el resultado de un proceso por medio del cual cada área conoce su función de manera específica, por lo que es un diseño que conceptualiza las competencias AGLALA ISNN 2215-7360

2014; 5 (1): 126-142 
especializadas que deben de ejecutar los trabajadores, desde el nivel directivo hasta el nivel operativo. La administración reconoce, según Daft (2007), que para que una organización empresarial tenga éxito tiene que tener en cuenta aspectos como son: el buen clima laboral, que los trabajadores tengan oportunidad para el crecimiento, cumplir las leyes laborales, etc., Ante esto, es notorio la importancia que estas variables tienen al momento del desempeño laboral por parte de los trabajadores y que repercutirán en el servicio que se brinde al cliente. Más aún, en momentos cruciales en lo que se requiera cubrir una necesidad o conseguir un propósito específico, como lo puede ser el resolver de la mejor manera posible los requerimientos de los clientes para lograr su satisfacción y, para que esto se pueda dar, es imperativo poner en obra las competencias para poder atender de manera efectiva al cliente.

La atención al cliente guarda estrecha relación con el manejo del tiempo, debido a que es actualmente un factor decisivo para lograr la satisfacción del usuario, el cual debe de ser atendido a la mayor brevedad posible y, ante un problema, cualquier miembro de la organización deberá buscar la solución de tal forma que no se retire insatisfecho de la empresa.

Para Barra (Citado en Gallardo y Galván, 1993), la calidad es que el cliente reciba lo que realmente desee, sea esto un bien o un servicio de manera apropiada para su uso y, es fundamental, hacerlo de tal manera que cada tarea sea realizada de manera correcta de principio a fin. Es por esto que la calidad la una diferenciación cuantitativa y cualitativa con respecto a un atributo requerido; es más, la calidad da valor al cliente. La calidad es la consecuencia de un arduo esfuerzo cuyo fin primordial es satisfacer el deseo del consumidor.

Hay que tener presente que la satisfacción que un cliente tiene es un estado emotivo al finalizar su compra de un producto o servicio, tal como lo expresa Fernández (2004) al decir que la satisfacción es una consideración positiva sobre un elemento específico producto de una AGLALA ISNN 2215-7360

2014; 5 (1): 126-142 
experiencia de compra. Siguiendo ese orden de ideas, Kotler y Lanekeller en el año 2006, dicen que la satisfacción es influenciada por la percepción en la calidad y el precio de un bien o servicio. Es por ello que el juez de la calidad sobre un bien o servicio no es la empresa, sino el cliente y por ello debe de brindársele un servicio o producto de acorde con sus expectativas.

Fernández contempla tres elementos sobre la definición de calidad al cliente, los cuales son: Primero, excelencia en el diseño; es cuando el servicio no solo responde, sino que excede las expectativas de los clientes. Segundo, consistencia en la producción; que significa el grado de homogeneidad en la producción de un servicio a través del tiempo y por las distintas personas que lo ejecutan y, por último, relación con las expectativas de los clientes; que se enmarca en los que estos desean a lo largo del tiempo.

\section{Perfil del personal que atiende a los clientes}

El personal que va a tratar de manera directa con los clientes tiene una labor de gran importancia y responsabilidad. Debido a ello, debe de tener determinadas capacidades y aptitudes, rasgos de personalidad, intereses y motivaciones que lo convierta en apto para este cargo.

Dib (2004), presenta seis atributos del personal que atiende clientela, los cuales son: a) Verdadera vocación de servicio, ante el cual el empleado debe de gustarle su trabajo y sentirse muy a gusto, esto porque le brindarán a los clientes la confianza debida para que pueda expresar sus necesidades y poder ser ayudados. b) Capacidad para escuchar al cliente, por el cual el encargado debe de prestar atención debida a lo que el cliente exponga de manera verbal o gestual, para que se pueda interpretar el mensaje de lo que quiere que se le brinde. c) Empatía, esto es ponerse en el lugar del otro ya que de esa manera se puede comprender la necesidad que se requiere satisfacer.

AGLALA ISNN 2215-7360

2014; 5 (1): 126-142 
d) Simpatía natural, esto significa que no debe de faltar en el personal de atención el carisma, debido a que esto influye grandemente y el cliente pueda percibirlo a través de una buena atención y se sienta a gusto en el establecimiento. e) Paciencia, ecuanimidad y autocontrol, para ello el personal debe de saber actuar ante el cliente y controlarse para que el tal no perciba incomodidad al momento de ser atendido y f) Capacidad de adaptación, el personal debe de actuar de acuerdo a la situación que presente cada cliente, debido a que ellos no tienen las mismas características comportamentales.

Las organizaciones tienen que tomar muy en cuenta al talento humano que su cara visible; por lo tanto, si se centra la atención en el decisivo papel que tiene el personal de servicio y se desarrolla estrategias que busquen la prestación efectiva del servicio al cliente, la empresa será la gran beneficiada con clientes permanentes y se mantendrá en la vanguardia a pesar de la competencia. En palabras de Zeithmal y Bitner (2002): “en una institución de servicio, si no estás atendiendo al cliente más te vale atender a alguien que sí lo sea” (p. 349).

\section{Clima laboral y servicio al cliente}

El papel que juega el personal de servicios en la atención al cliente es de suma importancia. Un importante factor es el sentido de pertenencia de los trabajadores para con la organización, ya que si existe empleados satisfechos aumentará la posibilidad de que los clientes reciban un buen servicio.

En los últimos años la atención al cliente es fundamental y, debido a ello, el cliente interno es considerado como pieza clave para el crecimiento organizacional. El éxito del tal se obtendrá por tener un grupo sólido y compacto que busque para conseguir los objetivos de la empresa.

AGLALA ISNN 2215-7360

2014; 5 (1): 126-142 
Si una institución mantiene un buen clima laboral, se verá reflejado en sus trabajadores y se manifestará en una mayor motivación, comunicación fluida, relaciones grupales y al final, como consecuencia, una mejor calidad de vida para los colaboradores, así se sentirán comprometidos con la empresa y su compromiso crecerá, lo cual repercutirá en la buena imagen organizacional. Todo esto, afectará de manera directa o indirecta en la calidad del servicio al cliente, ya que toda persona que va a un establecimiento tiene expectativas del lugar donde va a comprar o consumir un bien o servicio, trayendo al final, una satisfacción. Por el contrario, si el personal no cubre las expectativas del cliente, éste se sentirá insatisfecho y no regresará más al negocio. Además, de que tal cliente utilizará el mercadeo boca a boca para disminuir la credibilidad corporativa lo cual traerá pérdidas en la empresa.

Las instituciones son organismos sociales y humanos, por lo que los trabajadores tienen que sentir que pertenecen a esa organización, que hacen una labor y una aportación de valor para la sociedad. No es de gran valor, el que una empresa brinde buenos productos a sus clientes sino se preocupa por satisfacer a sus clientes internos, que son al fin y al cabo, los que transmitirán un sentimiento positivo o negativo al momento de interactuar con el cliente. Si el tal logra percibir como los miembros de una entidad corporativa tienen una mala relación interpersonal y es conflictiva, esto dañará la imagen organizacional y puede ser razón de no volver más a tal lugar.

Se toma como ejemplo un rubro organizativo, como son las empresas de servicio del sector turístico, debido a su amplia y diversificación de actividades productivas que ofertan y son solicitadas por los usuarios, la cadena de servicio que componen a esta industria se da a través de las agencias de promoción y ventas, transporte, restaurantes, hoteles, sitios de interés turístico, tiendas de artesanías. Debido a ello, se necesita un constante replanteamiento de las funciones que deben desempeñar cada uno de los empleados para poder enfrentar con éxito los permanentes y AGLALA ISNN 2215-7360

2014; 5 (1): 126-142 
novedosos retos. El trabajo que se realiza en esta área empresarial es un 70\% práctico, cara a cara con el cliente, debido a que el trabajador trabaja en áreas de servicio directo como son los aeropuertos, agencias de viajes, restaurantes, hoteles, parques diversos. En Colombia, las personas que prestan este servicio son en mayoría empíricos, tal como lo expresa Cabarcos (2006), contrastando con los países más desarrollados, los cuales cuentan con personal con mayor preparación intelectual.

La calidad del servicio que se brinda a los clientes es el elemento productivo y comercial que se constituye como un verdadero factor diferenciador y de mayor valor agregado. Esto debido a que el sentido de servicio que va de la mano con un producto requiere del desarrollo de mejores competencias especializadas por parte de los trabajadores que forman parte de la cadena del servicio. En el turismo, el trabajador tiene la oportunidad de ofrecer una experiencia única al cliente que vaya más allá del elemento transaccional, por medio de crear una relación, y esto de una satisfacción óptima del cliente.

\section{Conclusiones}

Las competencias al servir al cliente deben de ser entendida como una estrategia en la que todos los niveles de la empresa estén directa o indirectamente comprometidas con los clientes. Esto se dará si todos los procesos se encaminan al logro de los resultados misionales y a que los individuos que forman parte de la empresa interioricen una vocación legítima hacia el servicio y se comprometan de manera directa con los resultados de la organización. Además, al momento de seleccionar al personal idóneo a un puesto en una empresa de servicio, se debe de tener en cuenta atributos personales que son elementos de personalidad que se presentan a través del respeto, la 
adaptabilidad, la recursividad, destreza y sensibilidad social, persuasión, habilidades de comunicación, etc.

El aprendizaje, desarrollo y fortalecimiento de las competencias en un aspecto clave y crítico dentro de la cultura del servicio. Esto demanda la capacitación de manera continua de todos los trabajadores, poniendo énfasis en los que tienen trato directo con el cliente, pues según su desempeño dependerá la satisfacción y la fidelización del cliente. Todo lo anterior, aunado a la motivación, disposición y entrenamiento del trabajador.

\section{Referencias bibliográficas}

Brunet, L. (1987). El clima de trabajo en las organizaciones: definición, diagnóstico y consecuencias. México: Trillas.

Brunet, L. (2007). El clima de trabajo en las organizaciones: definición, diagnóstico y consecuencias. México: Trillas.

Bustos, P., y Miranda, M. (2009). Clima organizacional. Santiago: Lautaro.

Cohen, Bruce J. (1992). Introducción a la sociología. México: McGraw Hill.

Cabarcos, N. (2006). Promoción y venta de servicios turísticos. Madrid: Editorial Ideas Propias.

Dib, A. (2004). El servicio al cliente, la venta y el marketing personal. Buenos Aires: Machi.

Daft, R. (2007). Teoría y diseño organizacional. México: Cencage Learning Editores.

Fernández, P. (2004). La gestión del marketing de servicios. Argentina: Mc. Graw Hill.

Gallardo, R. y Galván, B. (1993). El análisis de la relación existente entre la calidad en el servicio y satisfacción del consumidor de las tiendas de autoservicio. Tesis de Licenciatura. Instituto de Estudios Superiores de Tamaulipas.

Koenes, A. (1996). Diseño del servicio: paso a paso. España; Díaz de Santos S.A.

AGLALA ISNN 2215-7360

2014; 5 (1): 126-142 
Kolb, D., Rubin, I. y Mcintyre, J. (2001). Psicología de las organizaciones. México: Prentice Hall.

Kotler, P y Keller, K. (2006). Dirección de marketing. México: Mc Graw Hill

Pelaes, O. (2010). Relación entre el clima organizacional y la satisfacción del cliente en una empresa de servicios telefónicos. Tesis de doctorado. Universidad Nacional Mayor de San Marcos, Lima, Perú.

Pfeffer, J. (2006). Cómo mejorar el clima organizacional. Argentina: Universidad de Standford. Snow, J. (2003). Estimular el clima de trabajo para mejorar la actuación y retener a empleados estimados. Revista Jona, 32, 7-8

Shein, E. (1996). La cultura empresarial y el liderazgo. Barcelona: Plaza y Janés.

Seisdedos, N. (1996). El clima laboral y su medida. Psicología del trabajo y de las organizaciones.

Sandoval. M. (2004). Concepto y dimensiones del clima laboral. Revista Hitos de Ciencias Económico Administrativas, 10, 27, pp.83-87.

Tarazona, D. y Roger, A. (2004). Condiciones psicosociales del trabajo. Lima: UNMSM

Zeithmal, V. y Bitner, M. (2002). Marketing de servicios: Un enfoque de integración del cliente a la empresa. México: Mc Graw Hill

AGLALA ISNN 2215-7360

2014; 5 (1): 126-142 